\title{
Evaluation of Multiparametric MRI of the Prostate: Impact of Standardized Examination Protocols and the Endorectal Coil
}

\author{
Finn Rampoldt, Frank Huebner, Stephan Zangos, Andreas Bucher, Jan-Erik Scholtz, \\ Moritz Kaup, Sebastian Fischer, Thomas J. Vogl, Boris Bodelle \\ Department of Diagnostic and Interventional Radiology, Goethe University of Frankfurt, Frankfurt, Germany \\ Email: finnrampoldt@web.de
}

How to cite this paper: Rampoldt, F., Huebner, F., Zangos, S., Bucher, A., Scholtz, J.-E., Kaup, M., Fischer, S., Vogl, T.J. and Bodelle, B. (2020) Evaluation of Multiparametric MRI of the Prostate: Impact of Standardized Examination Protocols and the Endorectal Coil. Open Journal of Medical Imaging, 10, 30-41.

https://doi.org/10.4236/ojmi.2020.101003

Received: December 14, 2019

Accepted: February 18, 2020

Published: February 21, 2020

Copyright $\odot 2020$ by author(s) and Scientific Research Publishing Inc. This work is licensed under the Creative Commons Attribution International License (CC BY 4.0).

http://creativecommons.org/licenses/by/4.0/

\begin{abstract}
Purpose: The aim of the present study was to evaluate the patient population, different MRI examination protocols and the patient's acceptance of the endorectal coil in the diagnosis of prostate cancer regarding the current discussion in PI-RADS v2.1. Material and Methods: In our institute, 256 patients were examined with different protocols and separated into six groups. The value of the different MRI protocols was identified by analyzing sensitivity, specificity, negative predictive value and positive predictive value. The patient population was tested for statistically significant differences in their characteristics to detect a distortion of the results. The patients' acceptance of endorectal coil was evaluated by a query. Results: In total $4.7 \%$ of the patients would not recommend a MRI examination because of subjective physical strain and $65.6 \%$ of all patients subjectively saw a benefit in the examination in regard to an optimal diagnostic process. The protocol groups reached a sensitivity from $66.7 \%-100 \%$, a specificity from $40 \%-75 \%$, a positive predictive value from $33.3 \%-80 \%$ and a negative predictive value from $66.7 \%$ $100 \%$. Conclusion: MRI of the prostate is a safe and comfortable tool with high sensitivity and negative predictive value and the potential of ruling out a clinically significant prostate cancer. However, a general recommendation for the use of biparametric MRI could not be given, considering a higher sensitivity could be performed when using dynamic contrast-enhanced MRI or spectroscopy.
\end{abstract}

\section{Keywords}

Prostate Imaging, Prostate Cancer, Multiparametricmri 


\section{Introduction}

The importance of Magnet Resonance Imaging (MRI) in the diagnosis of prostate cancer is increasing rapidly. Because of the poor sensitivity and high number of side effects of transrectal ultrasound guided biopsy (TRUS-Bx) [1] [2] [3] [4] and the high sensitivity and negative predictive value of MRI [5] [6] [7], more and more patients are being examined by this method in case of supposed prostate cancer.

In 2012, the European Society of Urogenital Radiology (ESUR) developed guidelines for judging MRI examinations of the prostate and recommended the use of multiparametric MRI (mpMRI), containing T1 and T2 weighted images (T2w), diffusion weighted imaging (DWI), dynamic contrast enhanced images (DCE) and optional proton-magnetic-resonance-spectroscopy-imaging (H-MRSI) (Figures 1-4) [8]. Furthermore, recommendations for the minimal technical requirement and the evaluation of prostate MRI were given. Thus, the prostate imaging reporting and data system (PI-RADS) score have been developed. Several studies confirmed the use of these standardizations of MRI in the diagnosis of prostate cancer [9] [10], but it could be shown that the H-MRSI yields no further use in MRI of the prostate [9]. In 2014, the ESUR and the American College of Radiologists (ACR) revised the standardizations of MRI and developed PI-RADS v2 [11]. The achievement of H-MRSI was not recommended any more. In MRI for the diagnosis of prostate cancer, T2 weighted images pointed out as the dominant sequence for the evaluation of the central zone and the DWI for the peripheral zone of the prostate [11]. Additional dynamic contrast enhanced sequences (DCE) could be used in case of unclear findings. Further studies have shown promising results [12] [13] [14]. The latest modifications of the standardization of the MRI in the diagnosis of prostate cancer were published in 2019. In these PI-RADS v2.1 guidelines, the technical requirements and recommended MRI sequences were revised. Regarding time and cost-effectiveness, the use of biparametric MRI (bpMRI) consists of T2 weighted images and DWI, was discussed [15]. First studies proved the use of bpMRI on therapy naïve patients [16] [17]. The use of the endorectal coil in the diagnosis of prostate cancer is still unclear. The scientific literature shows contradictory results [18] [19] [20].

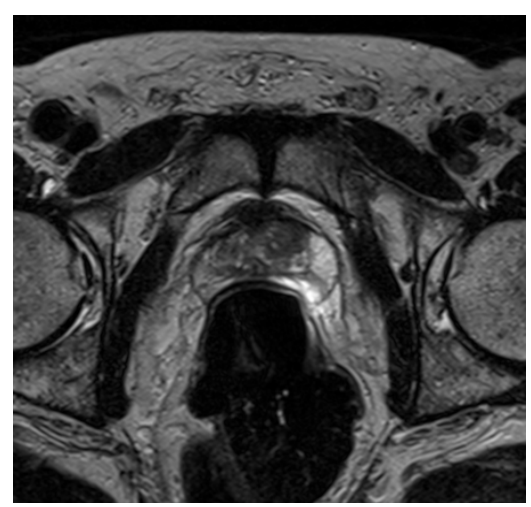

Figure $1 . \mathrm{T} 2$ weighted images (T2w). 


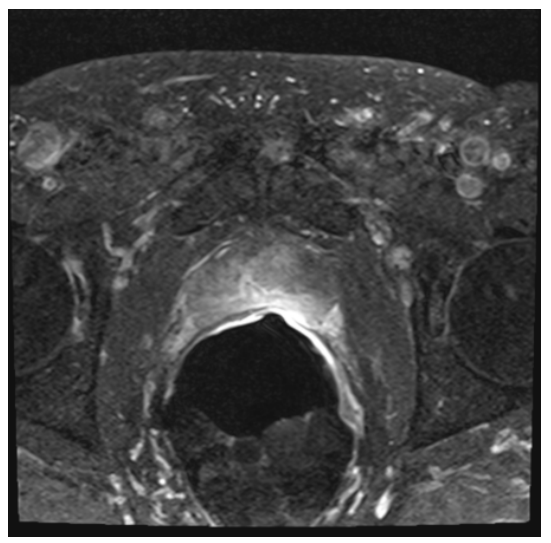

Figure 2. Dynamic contrast enhanced images (DCE).

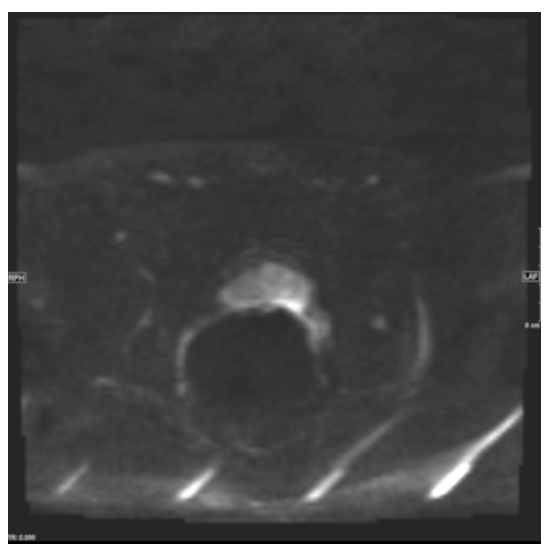

Figure 3. Diffusion weighted images (DWI).

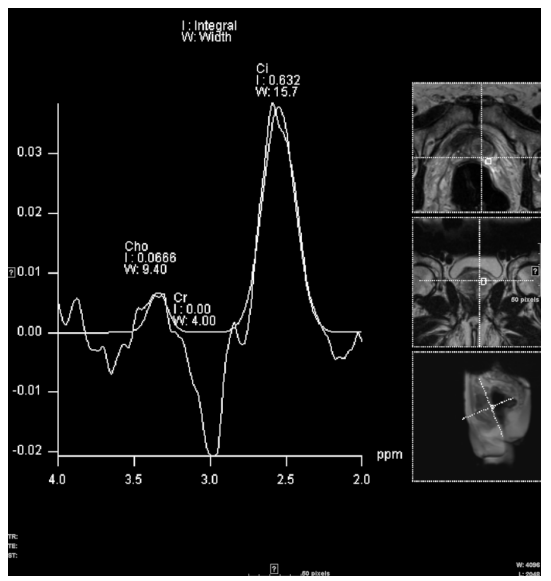

Figure 4. Proton magnetic resonance spectroscopy imaging (H-MRSI).

In our institute, we have been using MRI in the diagnosis of prostate cancer since January 2007, five years before the first guidelines of the ESUR were published. The aim of the present study was to evaluate the patients and the different examination protocols of our institute regarding the current discussion in PI-RADS v2.1 guidelines. For technical evaluation, the patients' acceptance of the endorectal coil was assessed. 


\section{Materials and Methods}

\subsection{Patients}

MRI was performed on 256 patients between January 2007 and December 2010. Patients with a suspicious Digital-rectal-Examination (DRE) and/or a Prostate-Specific-Antigen (PSA) level $>4 \mathrm{ng} / \mathrm{ml}$ were included in this study. This cut off value is recommended by the German Society of Urology [21] and common in the current literature [6] [22]. Exclusion criteria were general contraindications to MR imaging like heart pacemakers, allergy to contrast agents, severe claustrophobia, to biopsies and a known prostate cancer. Patient's age, PSA level, diagnosed cancer, therapy, previous biopsies (MR or TRUS-guided biopsy) and patient's medication were evaluated by a query. Further, the patients' acceptance of the endorectal coil was analyzed.

\subsection{Examination Technique}

For MRI examinations the scanner types Trio, Avanto and Espree of the firm Siemens with a field strength of $3 \mathrm{~T}$ were used. Additionally, we used an endorectal coil. All patients received biopsy after MRI. For MRI of the prostate T2 weighted images (T2w), diffusion weighted sequences (DWI), proton magnetic resonance spectroscopy (H-MRSI) and dynamic contrast enhanced sequences (DCE) were used. The results of the examinations were evaluated by a radiologist with 15 years of experience judging MRIs of the prostate.

\subsection{Subgroups}

Due to further developments of MRI protocols in our institution between 2007 and 2010, patients were examined with different protocols and separated into six groups accordingly (Table 1). The value of the different MRI protocols was identified by analyzing sensitivity, specificity, negative predictive value and positive predictive value.

\subsection{Statistics}

The patient population was tested for statistically significant differences in their characteristics to detect a distortion of the results by using the chi-square test. All statistical analyses were performed with dedicated software (BiAS 9.17, Epsilon, Frankfurt, Germany).

Table 1. MRI protocols with included sequences and patients.

\begin{tabular}{ccc}
\hline MRI Protocol & Included Sequences & Number of Patients \\
\hline Group A & T2 & 5 \\
Group B & T2, DWI & 17 \\
Group C & T2, DCE & 8 \\
Group D & T2, H-MRSI & 48 \\
Group E & T2, DCE, DWI & 3 \\
Group F & T2, DCE, DWI, H-MRSI & 47 \\
\hline
\end{tabular}




\subsection{IRB Approval}

IRB approval was issued by the ethics commission of the Goethe University of Frankfurt on the 26th of February 2016.

\section{Results}

\subsection{Patients}

After disposing of the exclusion criteria 128 patients with a mean age of 72 (range 52 to 93 ) and a mean PSA value of $6.92 \mathrm{ng} / \mathrm{ml}$ (range $0.02 \mathrm{ng} / \mathrm{ml}$ to 34 $\mathrm{ng} / \mathrm{ml}$ ) were evaluated (Table 2). Prostate cancer was found in patients with a PSA value of $0-4 \mathrm{ng} / \mathrm{ml}$ in $45 \%$ (5/11), with a PSA of $4-10 \mathrm{ng} / \mathrm{ml}$ in $44 \%$ (25/57) and with a PSA $>10 \mathrm{ng} / \mathrm{ml}$ in $40 \%$ (8/20). PSA was not determined in 40 patients. Prostate cancer was diagnosed in $42.2 \%$ (54/128) of the patients. $57.4 \%$ (31/54) of the patients underwent prostatectomy. A previous biopsy of the prostate was performed in 75\% (96/128) cases. A MRI-guided biopsy was accomplished in $31.3 \%$ (30/96) of the patients. Medications with potential distortion of the PSA value were taken in 5.5\% (7/128) cases. For subjective acceptance of the MRI examination we asked our patients about their experiences with the endorectal coil during the examination. The survey of the patients showed that $68.8 \%$ (88/128) did not feel uncomfortable because of the endorectal coil. In total 4.7\% $(6 / 128)$ of the patients would not recommend a MRI examination because of subjective physical strain and $65.6 \%(84 / 128)$ of all patients subjectively saw a benefit in the examination in regard to an optimal diagnostic process.

\subsection{Examination}

Carcinoma suspect lesions were shown in 68\% (87/128) of all MRI examinations. The tumor stage according to the TNM system is shown in Figure 5. The results of the MRI protocols are shown in 3.3.

\subsection{Subgroups}

Statistical analyses of the particular MRI protocols are shown in Table 3. In every protocol, except group B and D, we reached a sensitivity of $100 \%$. We also reached a negative predictive value of $100 \%$ in the groups $\mathrm{A}, \mathrm{C}$ and $\mathrm{F}$.

\subsection{Statistics}

In the statistical analysis, no significant link between the used MRI protocol and the patient's age $(\mathrm{p}=0.63)$ or their PSA value $(\mathrm{p}=0.21)$ could be determined.

Table 2. Included patients and inclusion criteria.

\begin{tabular}{ccc}
\hline $\begin{array}{c}\text { Patients with complied inclusion criteria } \\
\text { Patients after return of the query }\end{array}$ & 256 & \\
$\begin{array}{c}\text { Patients after exclusion of those with known prostate } \\
\text { cancer or inadequately filled query }\end{array}$ & 128 & - Suspicious DRE and/or \\
\end{tabular}


Table 3. Results of MRI examinations (in brackets: 95\% confidence interval).

\begin{tabular}{ccccc}
\hline MRI Protocol & Sensitivity & Specifity & $\begin{array}{c}\text { Positive Predictive } \\
\text { Value }\end{array}$ & $\begin{array}{c}\text { Negative Predictive } \\
\text { Value }\end{array}$ \\
\hline Group A & $100 \%$ & $75 \%$ & $50 \%$ & $100 \%$ \\
Group B & $(2.5 \%-100 \%)$ & $(19.4 \%-99.4 \%)$ & $(13.7 \%-87.8 \%)$ & $(29.2 \%-100 \%)$ \\
& $(22.3 \%-95.7 \%)$ & $(23.4 \%-83.3 \%)$ & $(13.7 \%-87.8 \%)$ & $(34.9 \%-96.8 \%)$ \\
Group C & $(39.8 \%-100 \%)$ & $(19.4 \%-99.4 \%)$ & $(28.4 \%-99.5 \%)$ & $(29.2 \%-100 \%)$ \\
& $80 \%$ & $43.48 \%$ & $60.61 \%$ & $66.67 \%$ \\
Group D & $(59.3 \%-93.2 \%)$ & $(23.2 \%-65.5 \%)$ & $(42.1 \%-77.1 \%)$ & $(38.4 \%-88.2 \%)$ \\
& $100 \%$ & $0 \%$ & $33.33 \%$ & $0 \%$ \\
Group E & $(2.5 \%-100 \%)$ & $(0 \%-84.2 \%)$ & $(15.8 \%-100 \%)$ & $(0 \%-100 \%)$ \\
& $100 \%$ & $40 \%$ & $48.57 \%$ & $100 \%$ \\
Group F & $(80.5 \%-100 \%)$ & $(22.7 \%-59.4 \%)$ & $(31.4 \%-66.0 \%)$ & $(73.5 \%-100 \%)$ \\
\hline
\end{tabular}

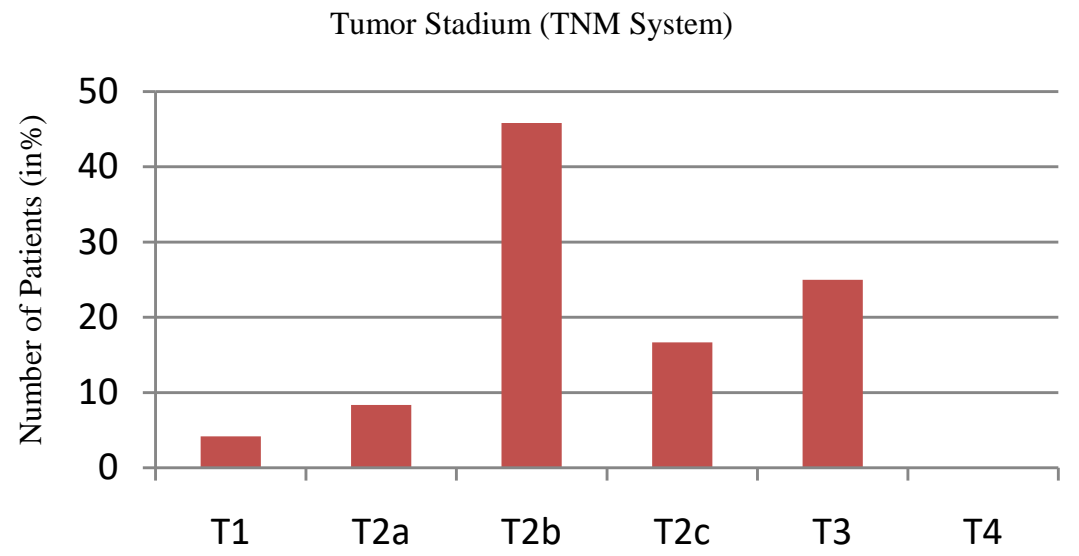

Figure 5. Tumor stadium according to the TNM system.

Additionally, we could not prove a significant amount of either patients diagnosed with prostate cancer or not in the protocol group $(\mathrm{p}=0.51)$. We could also find no relevant relation between the diagnosis of prostate cancer in the MRI findings and the protocol group $(\mathrm{p}=0.30)$. No significant correlation between the stage of the tumor (according to TNM classification) and the performed MR-sequences could be verified $(\mathrm{p}=0.80)$. A statistically significant number of prostate biopsies in one of the protocol groups could be ruled out $(\mathrm{p}=0.36)$. There was no statistical correlation between a diagnosed prostate cancer and the physical strain caused by the endorectal coil $(\mathrm{p}=0.22)$.

\section{Discussion}

\subsection{Other Groups Results}

The impact of multiparametric MRI has been evaluated in several studies and meta-analyzes. A sensitivity up to $96 \%$ and a specificity up to $88 \%$ have been reported [23] [24]. In 2017, Ahmed et al. postulated that a multiparametric MRI 
performed before the biopsy could avoid up to $27 \%$ of all biopsies of the prostate [7]. However, the precise MRI protocol is still unclear. As mentioned, in the latest PI-RADS v.2.1 guidelines a biparametric MRI is being discussed regarding time- and cost-effectiveness [15]. Furthermore, there is potential to stint contrast medium containing gadolinium. However further research documented the possible use of further MRI sequences, especially in the diagnosis of early staged prostate cancer [25] [26] [27]. Kim et al. recommended the use of multiparametric MRI in case of PI-RADS score 3 [28]. The benefit of the endorectal coil in the diagnosis of prostate cancer is still in discussion as well. In the current literature, Barth et al. could not find a benefit of the endorectal coil when using a $3 \mathrm{~T}$ tomograph, while Hoffner et al. as well as Mirak et al. indicated an advantage when evaluating the posterior and peripheral zone of the prostate [19] [20].

\subsection{Patients}

The use of a suitable patient population is an important precondition of a representative study. The participating patients showed similar epidemiological characteristics in age and PSA as patients of comparable studies [7] [23] [24]. The disposition of prostate cancer in the PSA groups in this study is striking. Compared to other epidemiological studies there is a high number of patients with a prostate cancer and a PSA value of $0-4 \mathrm{ng} / \mathrm{ml}$. With a percentage of $42.2 \%$ there is even a high number of prostate cancer in total. A possible reason for these findings is the patients' preselection with suspicious examinations like DRE or dedicated clinical findings. However, in 31.3\% (40/128) of all patients, we were not able to determine the PSA level. This could be a possible reason for a distortion of our results. Most of the patients with diagnosed prostate cancer underwent prostatectomy. Although the operation constitutes the gold standard in most cases, there is a high potential of side effects with a massive constraint of life quality [29] [30] [31].

A high number of patients underwent a previous prostate biopsy. But in only $31.3 \%$ of all cases, a MR-guided prostate biopsy has been performed. This reflects the small importance of MR-guided biopsy at this early stage of MRI of the prostate. Regarding the high number of side effects during the prostate biopsy [1] [2], it is an important aim to reduce the number of prostate biopsies in general. Current studies show that the MRI has high potential to reduce these biopsies [7] and is a feasible and safe tool to target prostate biopsy [22] [32]. There is also the possibility of the fusion of MR and sonography scans to get a better targeted biopsy. In several researches, this technique helps to reduce the number of biopsies up to 83\% [33] [34].

The use of the endorectal coil achieved a high patients' acceptance. Only $31 \%$ of the patients felt explicitly impaired by the coil. Regarding the inconsistent literature, we see this result as an argument for the use of the endorectal coil. However, this procedure is an invasive treatment with possible side effects. Thus non-invasive alternatives are definitely preferable, but would need further re- 
search before they could be recommended [35].

\subsection{Examinations}

In case of diagnosed prostate cancer, it is conspicuous that there was a very high number of $\mathrm{T} 2$ staged prostate cancer. The very low number of $\mathrm{T} 1$ cancer could be reasoned by the fact that there are still difficulties in detecting a small sized prostate cancer. Even in 2011, Röthke et al. postulated that the possibility of detecting prostate cancer in the MRI depends on its size [36]. Although there is no direct therapeutical consequence for $\mathrm{T} 1$ cancer in a lot of cases regarding the concept of watchful waiting, there is still a potential of improving the MRI of the prostate. According to the current literature the use of further sequences like H-MRSI could improve the detection rate of small sized prostate cancer [26].

\subsection{Subgroups}

The group A in our study was examined with only morphological sequences (Table 1 and Table 3). These at first sight impressive findings should be taken with great care because of the very low number of patients.

In our study, we had five groups with the combination of morphological and functional MRI sequences. The results are shown in Table 3.

Table 3 shows excellent findings in group $\mathrm{C}$ and group $\mathrm{F}$ compared with former findings. However, our results in group $\mathrm{C}$ were achieved with a very small number of patients and should be taken with great care. Compared with the results of the current studies there are at least two important points.

First, the multiparametic MRI did not reach the high specificity of similar studies, which means we had a higher number of false-positive findings. A possible reason is the fact, that our study group was examined between 2007 and 2010. Two years later the ESUR published recommendations for the examination and the judging of multiparametic MRI, like the PI-RADS score.

Second, we could confirm the excellent results regarding sensitivity and negative predictive value. Considering the results there is always a higher sensitivity and negative predictive value than in the urological standard diagnostics, including PSA, DRE and TRUS-guided biopsy, regardless of the used examination protocol.

Currently, the use of biparametric MRI is in discussion regarding time- and cost-effectiveness [15]. In our study, we could improve the sensitivity by adding an extra examination sequence, like DWI or H-MRSI. A general recommendation for the use of bpMRI could not be given by our results. However, further research is necessary because of the high socio-economic importance.

\subsection{Statistics}

There was no statistical correlation between the patient's acceptance of the endorectal coil and a diagnosed prostate cancer. A potential distortion of results could be ruled out. The statistical analyses of the subgroups yield no significant 
differences regarding several characteristics like age, PSA value, or diagnosed prostate cancer. A potential distortion of the results due to a random disposition of important characteristics in a particular subgroup could be ruled out.

\subsection{Limitations}

There are several limitations to our study that should be mentioned:

Due to the fact that the TRUS-controlled biopsy can lead to scarring in the area of the prostate, there is a danger of a false-positive result. Even in 2005, Scheenen et al. described the possibility of TRUS controlled biopsies causing susceptibility-artifacts through hemorrhages into the tissue of the prostate [37]. A sufficient wait of six weeks between a biopsy and a MRI examination is therefore important [37]. This fact is discussed in the current PI-RADS v2.1 guideline [15], however it should be considered in further studies.

Furthermore, our study shows some methodical weaknesses, like a nonexistent randomization of the participants in the individual protocol groups. The randomization only took place at the time of the retrospective analysis which led to a considerable difference in the amount of patients in the protocol groups. As a consequence, representative statements and statistically significant examinations were made impossible by the fact that several MRI protocols were carried out with only a small amount of patients.

\section{Conclusion}

In conclusion, MRI of the prostate is a safe and comfortable tool with high sensitivity, specificity and negative predictive value in the diagnosis of prostate cancer and a high potential in ruling out a clinical significant prostate cancer. However, there is an ongoing discussion about some aspects like the dedicated examination protocol or the benefit of the endorectal coil. A recommendation for the biparametric MRI consisting T2 and diffusion weighted images, as proposed from the ACR, could not be given regarding our study results that the use of DCE or H-MRSI could improve the sensitivity. Because of the high patient's acceptance of the endorectal coil, we recommend its use in $3 \mathrm{~T}$ MRI according to current studies [19] [20]. However, further research is needed to accomplish more evidence for the examination protocol and the endorectal coil.

\section{Conflicts of Interest}

The authors report no actual or potential conflict of interest in relation to this article.

\section{References}

[1] Rosario, D.J., Lane, J.A. and Metcalfe, C. (2012) Short Term Outcomes of Prostate Biopsy in Men Tested for Cancer by Prostate Specific Antigen. Prospective Evaluation within Protect Study. BMJ, 344, d7894. https://doi.org/10.1136/bmj.d7894

[2] Rodriguez, L.V. and Terris, M.K. (1998) Risks and Complications of Transrectal 
Ultrasound Guided Prostate Needle Biopsy. A Prospective Study and Review of the Literature. The Journal of Urology, 160, 2115-2120. https://doi.org/10.1016/S0022-5347(01)62255-9

[3] Norberg, M., Egevad, L. and Holmberg, L. (1997) The Sextant Protocol for Ultrasound-Guided Core Biopsies of the Prostate Underestimates the Presence of Cancer. Urology, 50, 562-566. https://doi.org/10.1016/S0090-4295(97)00306-3

[4] Djavan, B., Milani, S. and Remzi, M. (2005) Prostate Biopsy: Who, How and When. An Update. The Canadian Journal of Urology, 12, 44-48.

[5] Johnson, L.M., Turkbey, B., Figg, W.D. and Choyke, P.L. (2014) Multiparametic MRI in Prostate Cancer Management. Nature Reviews Clinical Oncology, 11, 346-353. https://doi.org/10.1038/nrclinonc.2014.69

[6] Lawrentschuk, N. and Fleshner, N. (2009) The Role of Magnetic Resonance Imaging in Targeting Prostate Cancer in Patients with Previous Negative Biopsies and Elevated Prostate-Specific Antigen Levels. BJU International, 103, 730-733.

https://doi.org/10.1111/j.1464-410X.2008.08205.x

[7] Ahmed, H.U., El-ShaterBosaily, A. and Brown, L.C. (2017) Diagnostic Accuracy of Multiparametric MRI and TRUS Biopsy in Prostate Cancer (PROMIS): A Paired Validating Confirmatory Study. The Lancet, 389, 815-822. https://doi.org/10.1016/S0140-6736(16)32401-1

[8] Barentsz, J.O., Richenberg, J. and Clements, R. (2012) ESUR Prostate MR Guidelines 2012. European Radiology, 22, 746-757.

https://doi.org/10.1007/s00330-011-2377-y

[9] Roethke, M.C., Kuru, T.H. and Schultze, S. (2014) Evaluation of the ESUR PI-RADS Scoring System for Multiparametric MRI of the Prostate with Targeted MR/TRUS Fusion-Guided Biopsy at 3.0 Tesla. European Radiology, 24, 344-352. https://doi.org/10.1007/s00330-013-3017-5

[10] Schimmoller, L., Quentin, M. and Arsov, C. (2014) MR-Sequences for Prostate Cancer Diagnostics: Validation Based on the PI-RADS Scoring System and Targeted MR-Guided In-Core Biopsy. European Radiology, 24, 2582-2589. https://doi.org/10.1007/s00330-014-3276-9

[11] American College of Radiology (2015) MR Prostate Imaging Reporting and Data System Version 2.0. https://www.acr.org/-/media/ACR/Files/RADS/Pi-RADS/PIRADS-V2.pdf

[12] Hassanzadeh, E., Glazer, D.I. and Dunne, R.M. (2017) Prostate Imaging Reporting and Data System Version 2 (PI-RADS v2): A Pictorial Review. Abdominal Radiology, 42, 278-289. https://doi.org/10.1007/s00261-016-0871-z

[13] Smith, C.P. and Türkbey, B. (2018) PI-RADS v2: Current Standing and Future Outlook. Turkish Journal of Urology, 44, 189-194.

https://doi.org/10.5152/tud.2018.12144

[14] Polanec, S., Helbich, T.H. and Bickel, H. (2016) Head-to-Head Comparison of PI-RADS v2 and PI-RADS v1. European Journal of Radiology, 85, 1125-1131. https://doi.org/10.1016/j.ejrad.2016.03.025

[15] American College of Radiology (2019) MR Prostate Imaging Reporting and Data System Version 2.1. https://www.acr.org/-/media/ACR/Files/RADS/Pi-RADS/PIRADS-V2-1.pdf?la=en

[16] Alabousi, M., Salameh, J.P. and Gusenbauer, K. (2019) Biparametric vs Multiparametric Prostate Magnetic Resonance Imaging for the Detection of Prostate Cancer in Treatment-Naïve Patients: A Diagnostic Test Accuracy Systematic Review and Meta-Analysis. BJU International, 124, 209-220. https://doi.org/10.1111/bju.14759 
[17] Jambor, I., Verho, J. and Ettala, O. (2019) Validation of IMPROD Biparametric MRI in Men with Clinically Suspected Prostate Cancer: A Prospective Multi-Institutional Trial. PLoS Medicine, 16, e1002813. https://doi.org/10.1371/journal.pmed.1002813

[18] Kim, B.S., Kim, T.H., Kwon, T.G. and Yoo, E.S. (2012) Comparison of Pelvic Phased-Array versus Endorectal Coil Magnetic Resonance Imaging at 3 Tesla for Local Staging of Prostate Cancer. Yonsei Medical Journal, 53, 550-556. https://doi.org/10.3349/ymj.2012.53.3.550

[19] Hoffner, M.K., Huebner, F. and Scholtz, J.E. (2016) Impact of an Endorectal Coil for 1H-Magnetic Resonance Spectroscopy of the Prostate at 3.0T in Comparison to 1.5T: Do We Need an Endorectal Coil? European Journal of Radiology, 85, 1432-1438. https://doi.org/10.1016/j.ejrad.2016.05.019

[20] Mirak, S.A., Shakeri, S. and Bajgiran, A.M. (2019) Three Tesla Multiparametric Magnetic Resonance Imaging: Comparison of Performance with and without Endorectal Coil for Prostate Cancer Detection, PI-RADS ${ }^{\mathrm{m}}$ Version 2 Category and Staging with Whole Mount Histopathology Correlation. The Journal of Radiology, 201, 496-502. https://doi.org/10.1016/j.juro.2018.09.054

[21] German Society of Urology (2019) Interdisciplinary Guideline for Screening, Diagnosis and Therapy of the Prostate Cancer. Version 5.1.

https://www.leitlinienprogramm-onkologie.de/fileadmin/user_upload/Downloads/ Leitlinien/Prostata_5_0/LL_Prostatakarzinom_Langversion_5.1.pdf

[22] Engehausen, D.G., Engelhard, K. and Schwab, S.A. (2012) Magnetic Resonance Image-Guided Biopsies with a High Detection Rate of Prostate Cancer. Scientific World Journal, 2012, Article ID: 975971. https://doi.org/10.1100/2012/975971

[23] De Rooij, M., Hamoen, E.H., Fütterer, J.J., Barentsz, J.O. and Rovers, M.M. (2014) Accuracy of Multiparametic MRI for Prostate Cancer Detection: A Meta-Analysis. American Journal of Roentgenology, 202, 343-351. https://doi.org/10.2214/AJR.13.11046

[24] Fütterer, J.J., Briganti, A. and De Visschere, P. (2015) Can Clinically Significant Prostate Cancer be Detected with Multiparametric Magnetic Resonance Imaging? A Systemic Review of the Literature. European Urology, 68, 1045-1053. https://doi.org/10.1016/j.eururo.2015.01.013

[25] Leapman, M.S., Wang, Z.J. and Behr, S.C. (2017) Impact of the Integration of Proton Magnetic Resonance Imaging Spectroscopy to PI-RADS 2 for Prediction of High Grade and High Stage Prostate Cancer. Radiologia Brasileira, 50, 299-307. https://doi.org/10.1590/0100-3984.2016.0117

[26] Yang, B., Liao, G.Q. and Wen, X.F. (2017) Nuclear Magnetic Resonance Spectroscopy as a New Approach for Improvement of Early Diagnosis and Risk Stratification of Prostate Cancer. Journal of Zhejiang University Science B, 18, 921-933. https://doi.org/10.1631/jzus.B1600441

[27] Gholizadeh, N., Greer, P.B. and Simpson, J. (2019) Supervised Risk Predictor of Central Gland Lesions in Prostate Cancer Using $1 \mathrm{H}$ MR Spectroscopic Imaging with Gradient Offset-Independent Adiabaticity Pulses. Journal of Magnetic Resonance Imaging, 50, 1926-1936. https://doi.org/10.1002/jmri.26803

[28] Kim, Y.J., Huh, J.S. and Park, K.K. (2019) Effectiveness of Bi-Parametric MR/US Fusion Biopsy for Detecting Clinically Significant Prostate Cancer in Prostate Biopsy Naïve Men. Yonsei Medical Journal, 60, 346-351.

https://doi.org/10.3349/ymj.2019.60.4.346

[29] Kim, S.P., Gross, C.P. and Nguyen, P.L. (2014) Perceptions of Active Surveillance and Treatment Recommendations for Low-Risk Prostate Cancer: Results from a 
National Survey of Radiation Oncologists and Urologists. Medical Care, 52, 579-585. https://doi.org/10.1097/MLR.0000000000000155

[30] Klotz, L., Zhang, L. and Lam, A. (2010) Clinical Results of Long-Term Follow Up of a Large, Active Surveillance Cohort with Localized Prostate Cancer. Journal of Clinical Oncology, 28, 126-131. https://doi.org/10.1200/JCO.2009.24.2180

[31] Alberts, B.D., Woldu, S.L. and Weinberg, A.C. (2014) Venous Thromboembolism after Major Urologic Oncology Surgery: A Focus on the Incidence and Timing of Thromboembolic Events after 27,445 Operations. Urology, 84, 799-804. https://doi.org/10.1016/j.urology.2014.05.055

[32] Bodelle, B., Nagy, N.N. and Schulz, B. (2013) 1.5-T Magnetic Resonance-Guided Transgluteal Biopsies of the Prostate in Patients with Clinically Suspected Prostate Cancer. Investigative Radiology, 48, 458-463.

https://doi.org/10.1097/RLI.0b013e31827c394b

[33] Franiel, T., Eckardt, N., Waginger, M. and Horstmann, M. (2014) Prostatakarzinom. Der Radiologe, 54, 491-508. https://doi.org/10.1007/s00117-013-2608-0

[34] Schilling, D., Kurosch, M. and Mager, R. (2013) Fusion Imaging in Urology: Combination of MRI and TRUS for Detection of Prostate Cancer. Urology, 52, 481-489. https://doi.org/10.1007/s00120-012-3096-y

[35] O’Donohoe, R.L., Dunne, R.M., Kimbrell, V. and Tempany, C.M. (2019) Prostate MRI Using an External Phased Array Wearable Pelvic Coil at 3T: Comparison with an Endorectal Coil. Abdominal Radiology, 44, 1062-1069.

https://doi.org/10.1007/s00261-018-1804-9

[36] Röthke, M.C., Lichy, M.P. and Jurgschat, L. (2011) Tumorsize Dependent Detection Rate of Endorektal MRI of Prostate Cancer: A Histopathologic Correlation with Whole-Mount Sections in 70 Patients with Prostate Cancer. European Journal of Radiology, 79, 189-195. https://doi.org/10.1016/j.ejrad.2010.01.014

[37] Scheenen, T.W., Gambarota, G. and Weiland, E. (2005) Optimal Timing for in Vivo $1 \mathrm{H}-\mathrm{MR}$ Spectroscopic Imaging of the Human Prostate at 3T. Magnetic Resonance in Medicine, 53, 1268-1274. https://doi.org/10.1002/mrm.20468 